\title{
Usages des fourrages ligneux et pratiques pastorales dans la communauté rurale de Téssékéré, Ferlo, Nord Sénégal
}

\author{
Amy Bakhoum ${ }^{1 *}$ Oumar Sarr ${ }^{1}$ Daouda Ngom ${ }^{1}$ \\ Sékouna Diatta ${ }^{1}$ Alexandre Ickowicz ${ }^{2,3}$
}

\begin{abstract}
Mots-clés
Arbre, utilisation multiple, fourrage, éleveur pastoral, enquête, changement climatique, Sénégal
\end{abstract}

Submitted: 31 January 2019

Accepted: 20 April 2020

Published: 23 September 2020

DOI: $10.19182 /$ remvt.31890

\section{Résumé}

La communauté rurale de Téssékéré est à $97 \%$ peule avec comme principale activité l'élevage extensif. La transhumance est pratiquée dans cette zone sur de petites ou grandes distances en fonction de la disponibilité du fourrage. Les enquêtes réalisées auprès des éleveurs de la localité ont permis d'identifier leurs pratiques pastorales et de recueillir leur perception sur l'état actuel de la végétation et son évolution, et les facteurs responsables de cet état. L'arbre est utilisé dans cette communauté dans I'alimentation humaine $(94,3 \%)$ et animale $(94,3 \%)$, dans la pharmacopée $(82,8 \%)$, et dans les usages domestiques comme bois de service $(28,6 \%)$ et de construction $(37,14 \%)$. Pour accéder aux différentes parties des arbres, les éleveurs pratiquent souvent la coupe ou l'élagage. Les espèces les plus exploitées sont Balanites aegyptiaca $(14,3 \%$ à $88,6 \%)$, Calotropis procera (14,3\% à 28,6\%), Sclerocarya birrea $(8,6 \%$ à $25,7 \%)$, Adansonia digitata (8,6 \% à $20 \%$ ) selon les zones et les besoins. Une majorité d'éleveurs (51,4\%), conscients aujourd'hui de l'impact de ces pratiques sur le peuplement ligneux, a abandonné la coupe et pratique l'abaissement des branches pour nourrir le bétail.

- Comment citer cet article : Bakhoum A., Sarr O., Ngom D., Diatta S., Ickowicz A., 2020. Woody fodder uses and pastoral practices in the rural community of Tessekere, Ferlo, Northern Senegal. Rev. Elev. Med. Vet. Pays Trop., 73 (3): 191-198, doi: 10.19182/remvt.31890

\section{INTRODUCTION}

L'élevage en milieu sahélien est un élevage du type extensif. En milieu pastoral, l'alimentation en fourrage pendant la saison sèche ou en année de faible pluviométrie s'avère difficile avec les plantes annuelles, et dans ces cas le pâturage ligneux devient un élément important. L'économie de la plupart des pays sahéliens est basée sur l'exploitation des plantes ligneuses qui fournissent le pâturage naturel pour l'alimentation du bétail (Niang, 2009) et permettent aux populations rurales de subvenir à leurs besoins (Lykke, 2000).

\footnotetext{
1. Laboratoire d'écologie et d'éco hydrologie végétale (LEVEH), département de biologie végétale, FST/UCAD, BP 5005, Dakar-Fann, Sénégal. 2. CIRAD, UMR SELMET, F-34398 Montpellier, France.

3. SELMET, Univ Montpellier, CIHEAM-IAMM, CIRAD, INRAE, Institut Agro, Montpellier, France.

* Auteur pour la correspondance

Email : amybakhoum@hotmail.com
}

Les éleveurs, pour faire paître et abreuver le bétail en saison sèche, sont obligés de pratiquer la transhumance. La mobilité organisée des hommes et des troupeaux indispensables à la valorisation durable de ces écosystèmes par le pastoralisme (Benkhe et al., 1993) est une stratégie de base pour s'adapter à la forte variabilité spatio-temporelle des ressources pastorales. En effet, la satisfaction des besoins alimentaires du bétail est tributaire de la pluviométrie dont dépend directement la productivité des pâturages accessibles aux troupeaux (Banoin et Jouve, 2000). Cette pratique très courante permet aux éleveurs d'avoir un aperçu sur l'état de la ressource fourragère (composition, répartition spatiale et évolution). Ils disposent également de connaissances empiriques sur les plantes qui sont utilisées à des fins de pharmacopée traditionnelle mais aussi dans l'alimentation humaine et animale.

La végétation naturelle est pour l'homme un élément capital car elle peut subvenir aux multiples besoins d'une population à fort taux de croissance (Fontes et Guinko, 1995). Soubeiga (2004) ajoute que l'utilisation des produits forestiers non ligneux est d'un apport inestimable pour l'alimentation en milieu rural. Cependant, la surexploitation des 
espèces végétales et les mauvaises pratiques (Betti, 2001 ; Ouédraogo, 2008 ; Traoré 2008) combinées à la péjoration climatique (Diouf, 2002) sont à l'origine de la menace de disparition de certaines espèces.

Cet article présente une partie des résultats de la thèse d'A. Bakhoum (2013). L'objectif a été d'identifier les différentes pratiques pastorales les plus usitées ainsi que d'analyser la perception des éleveurs sur l'état actuel des ressources ligneuses et leur évolution spatio-temporelle.

\section{MATERIEL ET METHODES}

L'étude a été menée dans la communauté rurale (communalisation intégrale avec l'acte III de la décentralisation) de Téssékéré (14 37 à $16^{\circ} 50 \mathrm{~N}$ et $12^{\circ} 56$ à $16^{\circ} 26 \mathrm{O}$ ), dans le département de Linguère, dans la zone sylvopastorale. Des enquêtes ont été menées à l'aide d'un questionnaire individuel. Des campements ont été choisis aléatoirement. Compte tenu de la disponibilité des éleveurs dont certains étaient déjà en transhumance, nous n'avons pas eu l'occasion de questionner tous les éleveurs recensés. Au total 35 éleveurs ont été enquêtés dont 20 à Widou, 9 à Amaly et 6 à Téssékéré. Les informations recherchées étaient relatives à la perception des éleveurs sur l'état actuel des ressources, leur évolution dans le temps, les espèces disparues, celles en voie de disparition, ainsi que les raisons de leur disparition et de leur régression. Nous entendons par espèces disparues ou en voie de disparition celles qui ont existé avant la sécheresse de 1973 au niveau de la communauté et qui aujourd'hui n'y sont plus rencontrées. Il s'agissait également d'identifier les différentes pratiques pastorales, à savoir les usages des arbres se trouvant dans la communauté.

Un dépouillement manuel des questionnaires a été effectué, suivi d'une saisie des réponses à l'aide du logiciel Sphinx 4.5 qui génère directement les résultats. Le traitement des données a été effectué avec le tableur Excel 2007. Nous avons ensuite évalué la valeur usuelle (UV) des espèces pour chaque catégorie d'usage afin de montrer l'importance que la population accorde à une espèce donnée dans la localité (Sarr et al., 2013). Elle est obtenue par la formule $U V=\Sigma U / N$ où $U$ est le nombre de fois qu'une espèce est citée pour une catégorie d'usage, et $N$ le nombre total d'enquêtés.

\section{RESULTATS}

Les enquêtes ont montré que l'élevage était principalement pratiqué par les Peuls (97 \%), les autres éleveurs étaient des Maures. D'après nos résultats, $80 \%$ des enquêtés ont indiqué avoir hérité de cette activité de leurs parents. Le troupeau était constitué de bovins, d'ovins, de caprins et d'équins. A cette activité principale, les éleveurs en associaient souvent d'autres comme l'agriculture $(25,7 \%)$, le commerce $(31,4 \%)$, le jardinage $(3 \%)$ et le reboisement $(3 \%)$.

\section{Pratiques pastorales}

\section{Transhumance}

Le choix des itinéraires de transhumance était dicté par la disponibilité du fourrage mais surtout par l'accès à l'eau. Si pour certains les destinations étaient précises $(20 \%)$ pour la majorité la transhumance se faisait au hasard. A Téssékéré, les déplacements se faisaient sur de petites distances $(9,4 \%)$, sur de longues distances $(15,6 \%)$ et sur de petites et longues distances (75\%).

\section{Utilisation des plantes ligneuses}

\section{- Alimentation}

Les espèces les plus appréciées dans l'alimentation humaine sont consignées dans le tableau I. Les produits les plus utilisés (feuilles ou fruits) provenaient de Balanites aegyptiaca (88,6 \%), Adansonia

\section{Tableau I}

Espèces utilisées dans I'alimentation du bétail et humaine, et parties utilisées selon l'espèce dans I'alimentation humaine, communauté rurale de Téssékéré, Sénégal

\begin{tabular}{|c|c|c|c|c|c|}
\hline \multirow[t]{3}{*}{ Espèce } & \multicolumn{5}{|c|}{ Alimentation } \\
\hline & \multirow{2}{*}{$\begin{array}{c}\text { bétail } \\
\text { FC }\left(\%{ }^{\circ}\right)\end{array}$} & \multicolumn{4}{|c|}{ humaine } \\
\hline & & FC $\left(\%^{\circ}\right)$ & $\mathrm{Fe}$ & $\mathrm{Fr}$ & Ti Ec \\
\hline Balanites aegyptiaca & 65,7 & 88,6 & & * & \\
\hline Calotropis procera & 28,6 & & & & \\
\hline Sclerocarya birrea & 25,7 & 8,6 & & $*$ & \\
\hline Acacia raddiana & 20,0 & & & & \\
\hline Acacia seyal & 14,3 & 2,9 & & & $*$ \\
\hline Adansonia digitata & 14,3 & 20,0 & $*$ & $*$ & \\
\hline Ziziphus mauritiana & 11,4 & 11,4 & & $*$ & \\
\hline Boscia senegalensis & 5,7 & 2,9 & & $*$ & \\
\hline Grewia bicolor & 5,7 & 8,6 & $*$ & $*$ & \\
\hline Guiera senegalensis & 2,9 & & & & \\
\hline Lannea acida & 2,9 & & & & \\
\hline Terminalia avicennioides & 2,9 & & & & \\
\hline Piliostigma reticulatum & 2,9 & & & & \\
\hline Feretia apodanthera & 2,9 & & & & \\
\hline Sterculia setigera & 2,9 & 2,9 & & & $*$ \\
\hline
\end{tabular}

FC : fréquence de citation ; Fe : feuille ; Fr : fruit ; Ti : tige ; Ec : écorce

digitata (20\%), Ziziphus mauritiana (11,4\%), Grewiabicolor (8,6\%), et Sclerocarya birrea $(8,6 \%)$. Les fruits ou feuilles de Ba. aegyptiaca, Ad. digitata et Zi. mauritiana étaient autoconsommés (80\%) ou destinés à la vente (20\%). Les feuilles séchées et moulues de $A d$. digitata produisaient une poudre qui était mélangée au couscous, aliment de base des Peuls. Les fruits, communément appelés pain de singe, servaient à accompagner les bouillies de mil. Ba. aegyptiaca, l'espèce la plus citée était très présente dans la communauté (37,6 \%). Les espèces devenues rares (Acacia seyal et Sterculia setigera) dans la localité ont été peu fréquemment citées (2,9\%). La consommation d'une espèce devait dépendre de sa disponibilité.

Dans l'alimentation des animaux (tableau I), Ba. aegyptiaca, Calotropis procera, Sc. birrea et Acacia raddiana ont été les plus utilisées. Les enquêtes ont montré que le fourrage aérien était consommé directement par les animaux (94,3\%), ou mis à leur disposition par l'éleveur par émondage ou abaissement des branches pour qu'ils puissent manger $(5,7 \%)$. La majorité $(80 \%)$ des enquêtés a déclaré que les espèces étaient sollicitées du fait de leur disponibilité mais également pour leur qualité fourragère.

\section{- Pharmacopée}

Dans la communauté 14 espèces (tableau II) ont été citées pour l'utilisation de l'arbre dans la pharmacopée traditionnelle et parmi les espèces utilisées se trouvaient Ba. aegyptiaca (60\%), Sc. birrea (17,1\%) et Ca. procera (14,3\%). Les maladies traitées étaient l'hypotension, le diabète, le rhume, l'ulcère, les bouffées de chaleur et des maladies « surnaturelles ». Les organes utilisés étaient les fruits, l'écorce, les racines, les feuilles, et très rarement la sève par coupe et/ ou écorçage (figure 1). Le nom des organes des plantes qui étaient utilisés pour traiter les maladies surnaturelles n'a pas été communiqué par les éleveurs car ils étaient traditionnellement légués aux descendants. 


\section{Tableau II}

Espèces utilisées dans la pharmacopée, communauté rurale de Téssékéré, Sénégal

\begin{tabular}{|c|c|c|c|c|c|c|c|}
\hline \multirow[t]{2}{*}{ Espèce } & \multirow{2}{*}{$\begin{array}{c}\text { FC } \\
(\%)\end{array}$} & \multirow[t]{2}{*}{ Maladie traitée } & \multicolumn{5}{|c|}{ Partie utilisée } \\
\hline & & & Feuille & Ecorce & Racine & Fruit & Sève \\
\hline Acacia seyal & 2,9 & Diarrhée & & & & $*$ & \\
\hline Adansonia digitata & 8,6 & Diarrhée & & & & $*$ & \\
\hline Balanites aegyptiaca & 60,0 & Tension, estomac & & & & * & \\
\hline Boscia senegalensis & 5,7 & Bouffées de chaleur & & & * & & \\
\hline Calotropis procera & 14,3 & Fatigue, mal de dents & * & & * & & $*$ \\
\hline Commiphora africana & 2,9 & Maladie surnaturelle & & & & & \\
\hline Grewia bicolor & 5,7 & Bouffées de chaleur & * & & * & & \\
\hline Guiera senegalensis & 2,9 & Rhume & & * & & * & \\
\hline Lannea acida & 2,9 & Maladie surnaturelle & & & & & \\
\hline Mitragyna inermis & 2,9 & Maladie surnaturelle & & & & & \\
\hline Piliostigma reticulatum & 2,9 & Maladie surnaturelle & & & & * & \\
\hline Sclerocarya birrea & 17,1 & Diabète, estomac & & * & & * & \\
\hline Terminalia avicennioides & 2,9 & Maladie surnaturelle & & & & & \\
\hline Ziziphus mauritiana & 2,9 & Maladie surnaturelle & & & & & \\
\hline
\end{tabular}

$\mathrm{FC}$ : fréquence de citation
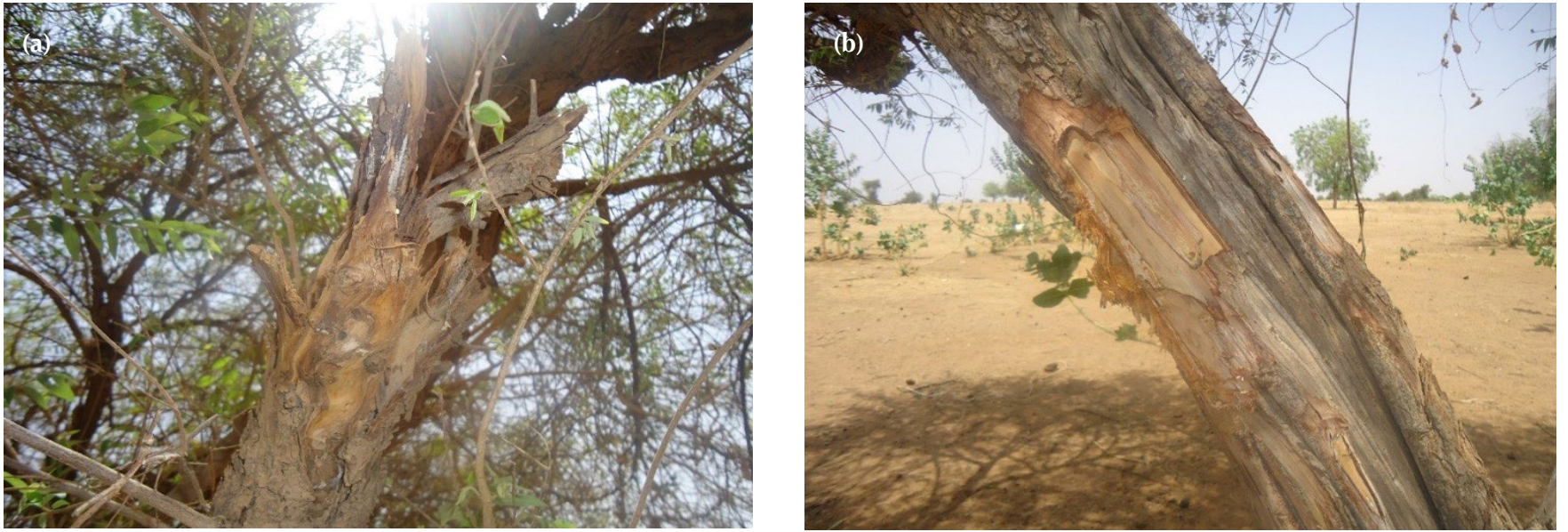

Figure 1 : coupe (a) et écorçage (b) de Grewia bicolor (Bakhoum, juin 2011).

- Usages domestiques

Le tableau III et la figure 2 montrent l'utilisation à des fins domestiques des espèces ligneuses. Ba. aegyptiaca et $\mathrm{Ca}$. procera étaient les plus utilisées. Cependant, respectivement $62,9 \%$ et $71,4 \%$ des enquêtés n'ont pas répondu à la question par rapport à leur utilisation comme bois de construction et bois de chauffage. A défaut les éleveurs utilisaient d'autres espèces ou des pieds morts.

\section{Perceptions des éleveurs}

Les éleveurs connaissaient la répartition spatiotemporelle des espèces végétales des pâturages que leurs troupeaux exploitaient.

\section{Répartition des espèces selon la topographie}

Le tableau IV montre la répartition de 24 espèces ligneuses selon la topographie du terrain. Parmi les espèces citées, 70,8 \%, 66,6 \% et 45,8\% étaient respectivement rencontrées au niveau des bas-fonds, des terrains plats et des dunes. Dans les bas-fonds, les espèces les plus présentes étaient Balanites aegyptiaca (51\%) et Sc. birrea (43\%), Mitragyna inermis (23\%) et Ad. digitata (20\%). Dans les terrains

\section{Tableau III}

Fréquence de citation des espèces utilisées pour le bois de chauffage ou de construction, communauté rurale de Téssékéré, Sénégal

\begin{tabular}{lcc|}
\hline Espèce & \multicolumn{2}{c|}{ Fréquence de citation (\%) } \\
\cline { 2 - 3 } & Bois de construction & Bois de chauffage \\
\hline Sans réponse & 62,9 & 71,4 \\
Calotropis procera & 22,9 & 14,3 \\
Balanites aegyptiaca & 14,3 & 8,6 \\
Grewia bicolor & 2,9 & 0 \\
Guiera senegalensis & 2,9 & 0 \\
Acacia raddiana & 0 & 2,9 \\
Ziziphus mauritiana & 0 & 2,9 \\
Acacia seyal & 0 & 2,9 \\
Arbre mort & 2,9 & 0 \\
Tout arbre & 0 & 2,9
\end{tabular}




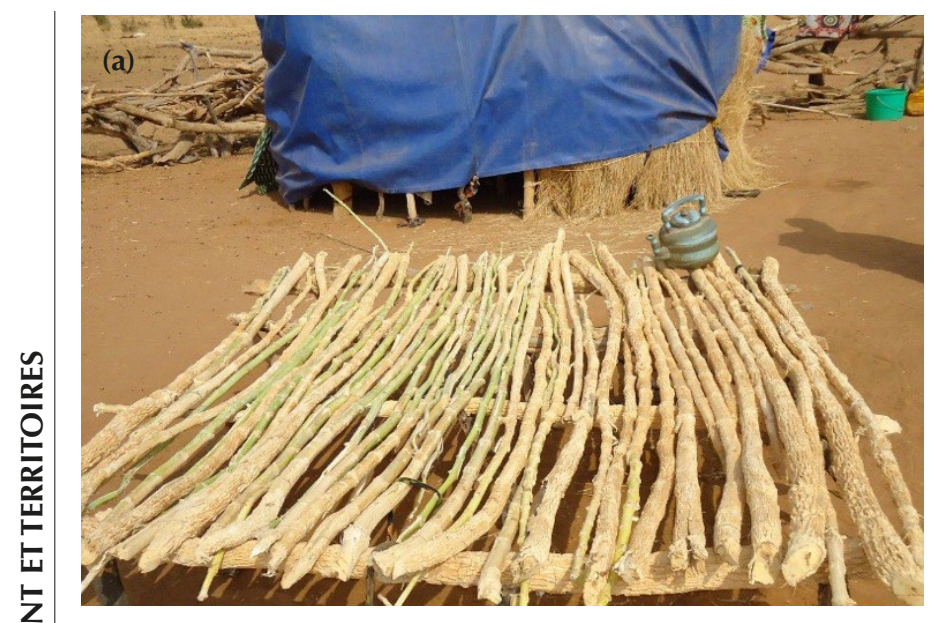

(b)

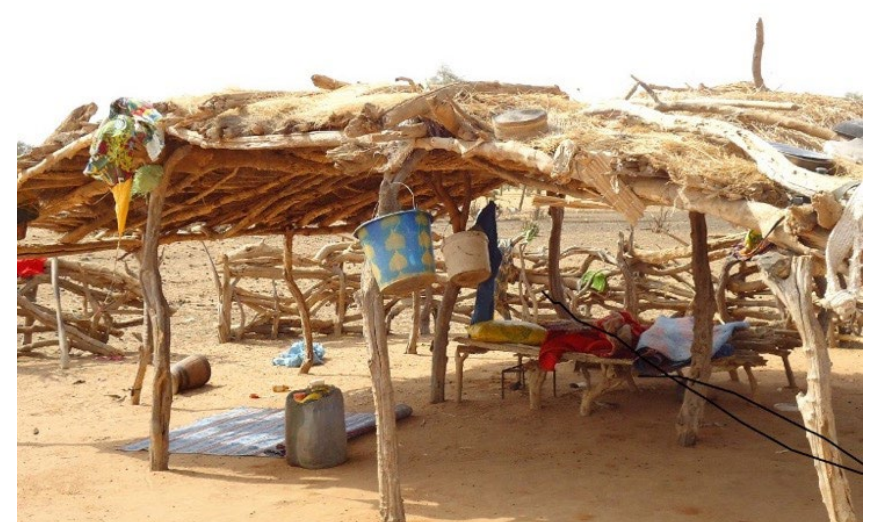

Figure 2 : branches de Calotropis procera en séchage pour construction (a) et ombriére (b) construite avec des pieds d'arbres morts et des branches de Calotropis procera (Bakhoum, janvier 2010).

\section{Tableau IV}

Répartition topographique des espèces selon leur fréquence de citation, communauté rurale de Téssékéré, Sénégal

\begin{tabular}{|c|c|c|c|}
\hline \multirow[b]{2}{*}{ Espèce } & \multicolumn{3}{|c|}{ Fréquence de citation ( $\%$ ) } \\
\hline & Bas-fond & Dune & Terrain plat \\
\hline Acacia nilotica & 6 & - & 11 \\
\hline Acacia pennata & 3 & - & - \\
\hline Acacia raddiana & 14 & 11 & 29 \\
\hline Acacia senegal & 11 & - & 11 \\
\hline Acacia seyal & 9 & - & 9 \\
\hline Adansonia digitata & 20 & - & 23 \\
\hline Anogeissus leiocarpus & 6 & - & - \\
\hline Balanites aegyptiaca & 51 & 17 & 94 \\
\hline Boscia angustifolia & 6 & - & - \\
\hline Boscia senegalensis & 3 & 6 & 34 \\
\hline Calotropis procera & 17 & 6 & 26 \\
\hline Combretum glutinosum & 3 & 6 & 6 \\
\hline Feretia apodanthera & 11 & - & - \\
\hline Gossypium barbadense & - & - & 9 \\
\hline Grewia bicolor & - & 26 & - \\
\hline Guiera senegalensis & 9 & 3 & 6 \\
\hline Leptadenia hastata & - & 3 & - \\
\hline Leptadenia pyrotechnica & - & 3 & - \\
\hline Maytenus senegalensis & - & 6 & 3 \\
\hline Mitragyna inermis & 23 & - & - \\
\hline Sclerocarya birrea & 43 & 14 & 91 \\
\hline Sterculia setigera & - & - & 3 \\
\hline Terminalia avicennioides & - & - & 3 \\
\hline Ziziphus mauritiana & 11 & - & 6 \\
\hline
\end{tabular}

plats, c'étaient Ba. aegyptiaca (94\%), Sc. birrea (91\%), Ac. raddiana (29\%) et Ca. procera (26\%). Pour les dunes, les fréquences de citation ont aussi été faibles en raison de la faible densité de végétation. Grewia bicolor (26\%) a été l'espèce la plus citée. Elle était suivie de Ba. aegyptiaca (17\%), Sc. birrea $(14 \%)$ et Ac. raddiana $(11,45 \%)$. Les espèces ubiquistes étaient Ac. raddiana, Ba. aegyptiaca, Ca. procera, Combretum glutinosum, Guiera senegalensis et Sc. birrea. Selon les besoins (fruits, feuilles, écorce...), les éleveurs pratiquaient plusieurs méthodes pour accéder aux parties sollicitées de la plante. Ils avaient recours au ramassage $(42,9 \%)$, à la coupe $(22,9 \%)$, à l'ébranchage (20\%), à l'effeuillage (28,6\%), à l'émondage (5,7\%), à la taille $(5,7 \%)$, à l'écorçage $(2,9 \%)$ et à la cueillette $(5,7 \%)$.

\section{Espèces disparues ou en voie de disparition}

Parmi les enquêtés 91,4 \% ont souligné la disparition de 31 espèces au niveau de Téssékéré (tableau V). Ces espèces se regroupent dans 13 familles botaniques et 24 genres. Au niveau des stations de Amaly, Téssékéré et Widou, 19, 13 et 27 espèces ont été respectivement citées. Ces espèces se répartissaient à Amaly dans 11 familles et 16 genres, à Téssékéré dans 8 familles et 10 genres, et à Widou dans 12 familles et 22 genres. La menace de disparition des arbres était donc plus perceptible à Widou, mais la famille la plus menacée de disparition aussi bien dans la communauté rurale qu'au niveau des trois stations était les Mimosaceae avec le genre Acacia.

Pour 88,6\% des éleveurs enquêtés, la disparition de ces arbres était principalement due à la rareté des pluies. Des moments repères ont été incriminés. Il s'agissait principalement des périodes de sécheresse de 1973, 1983 et 1991 qu'ils appelaient respectivement kitané bondé, fattar et fatar événement. Conscients aujourd'hui de l'état actuel des ligneux, 51,4 \% des enquêtés ont reconnu avoir changé ou abandonné des pratiques au cours du temps. Les pratiques abandonnées étaient la coupe $(45,7 \%)$, l'émondage (11,4\%), l'ébranchage $(5,7 \%)$, l'élagage $(2,9 \%)$ et les feux de brousse $(2,9 \%)$, au profit de l'abaissement des branches pour nourrir le bétail (figure 3). Outre la disparition d'espèces, les éleveurs ont également noté des changements au niveau de la densité et du recouvrement des espèces mais aussi de la structure de la végétation. Selon eux, les changements étaient imputables à la mauvaise pluviométrie $(85,7 \%)$, à la charge pastorale $(17,1 \%)$, à la croissance démographique $(8,6 \%)$ et aux feux de brousse $(8,6 \%)$.

\section{Evolution de la densité et de la production de fourrage aérien}

L'évolution de la fréquence spécifique des espèces et de leur production a été aussi abordée avec les éleveurs dans les trois stations (Amaly, Téssékéré et Widou). Parmi les 17 espèces citées à Amaly, les éleveurs à l'unanimité ont trouvé que la fréquence avait diminué pour toutes les espèces à l'exception de Ba. aegyptiaca et Sc. birrea dont les productions fourragères (feuilles et fruits) avaient également augmenté. Cependant, ils ont observé une diminution du nombre de Ad. digitata, Boscia senegalensis, Ca. procera et une amélioration 


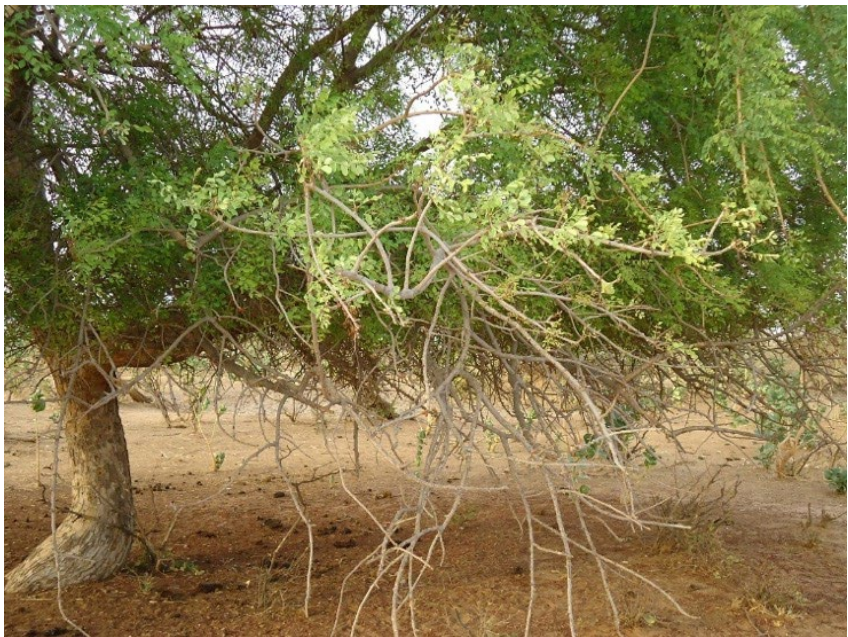

Figure 3 : abaissement des branches de Sclerocarya birrea pour le bétail (Bakhoum, janvier 2010).

\section{Tableau V}

Espèces en voie de disparition à Amaly, Téssékéré et Widou au Sénégal

\begin{tabular}{|c|c|c|c|c|}
\hline Famille & Espèce & Amaly & Téssékéré & Widou \\
\hline Anacardiaceae & Lannea acida & $d$ & $d$ & $d$ \\
\hline \multirow[t]{2}{*}{ Asclepiadaceae } & Calotropis procera & $d$ & $d$ & $d$ \\
\hline & Leptadenia hastata & 0 & 0 & $d$ \\
\hline Bignoniaceae & $\begin{array}{l}\text { Stereospermum } \\
\text { kunthianum }\end{array}$ & $d$ & 0 & $d$ \\
\hline \multirow[t]{2}{*}{ Bombacaceae } & Adansonia digitata & 0 & $d$ & $d$ \\
\hline & Bombax costatum & $d$ & $d$ & $d$ \\
\hline Burseraceae & Commiphora africana & $d$ & $d$ & $d$ \\
\hline \multirow[t]{4}{*}{ Caesalpiniaceae } & Bauhinia rufescens & $d$ & 0 & 0 \\
\hline & Piliostigma thonningii & $d$ & 0 & 0 \\
\hline & Pterocarpus erinaceus & $d$ & 0 & $d$ \\
\hline & Pterocarpus lucens & 0 & $d$ & 0 \\
\hline \multirow[t]{2}{*}{ Capparidaceae } & Boscia senegalensis & 0 & 0 & $d$ \\
\hline & Capparis tomentosa & 0 & 0 & $d$ \\
\hline Celastraceae & Maytenus senegalensis & 0 & 0 & $d$ \\
\hline \multirow[t]{6}{*}{ Combretaceae } & Anogeissus leiocarpus & 0 & 0 & $d$ \\
\hline & Combretum aculeatum & $d$ & 0 & $d$ \\
\hline & Combretum glutinosum & 0 & 0 & $d$ \\
\hline & $\begin{array}{l}\text { Combretum } \\
\text { micranthum }\end{array}$ & 0 & 0 & $d$ \\
\hline & Guiera senegalensis & $d$ & 0 & $d$ \\
\hline & Terminalia avicennioides & $d$ & $d$ & $d$ \\
\hline \multirow[t]{6}{*}{ Mimosaceae } & Acacia macrostachya & $d$ & $d$ & $d$ \\
\hline & Acacia nilotica & $d$ & $d$ & 0 \\
\hline & Acacia raddiana & 0 & 0 & $d$ \\
\hline & Acacia senegal & $d$ & $d$ & $d$ \\
\hline & Acacia seyal & $d$ & d & $d$ \\
\hline & Prosopis africana & 0 & 0 & $d$ \\
\hline Rhamnaceae & Ziziphus mauritiana & $d$ & 0 & $d$ \\
\hline \multirow[t]{3}{*}{ Sterculiaceae } & Feretia apodanthera & 0 & 0 & $d$ \\
\hline & Mitragyna inermis & $d$ & 0 & $d$ \\
\hline & Sterculia setigera & $d$ & $d$ & $d$ \\
\hline Tiliaceae & Grewia bicolor & $d$ & $d$ & $d$ \\
\hline
\end{tabular}

0 : non citée $; \mathrm{d}$ : citée disparue de leur production en feuilles et en fruits. Les fréquences de citation les plus élevées ont été obtenues avec Ba. aegyptiaca $(77,8 \%)$ et Sc. birrea $(33,8 \%)$ pour la production de feuilles et de fruits (tableau VI).

A Téssékéré (tableau VII) 16 espèces ont été citées par les éleveurs. A l'exception de Ba.aegyptiaca et $C a$.procera, tous les enquêtés ont trouvé que la densité de toutes les autres espèces citées avait diminué. Cependant, ils avaient des avis partagés concernant l'évolution des fréquences spécifiques de $\mathrm{Ba}$. aegyptiaca et $\mathrm{Ca}$. procera. Pour Ba. aegyptiaca, $50 \%$ des enquêtés ont observé une augmentation de la densité alors que 33,3\% ont noté une diminution. Pour $\mathrm{Ca}$. procera les mêmes proportions d'éleveurs ont affirmé que l'espèce avait diminué ou augmenté. La production de feuilles et de fruits de $A d$. digitata, Ba. aegyptiaca, Bo. senegalensis et Sc. birrea a augmenté de $16,7 \%$ à $66,7 \%$ selon les enquêtés.

A Widou (tableau VIII) 18 espèces ont été citées par les éleveurs. La perception des enquêtés par rapport à l'évolution de la densité des espèces Ac. raddiana, Ad. digitata, Ba. aegyptiaca, Boscia senegalensis, Ca. procera, Combretum aculeatum et Sc. birrea était contradictoire. Alors que $75 \%$ des enquêtés ont noté une augmentation du nombre d'individus de ces espèces, $25 \%$ ont attesté du contraire.

\section{Tableau VI}

Evolution de la densité et de la production de feuilles et de fruits selon les espèces à Amaly, Sénégal (1973 à 2010)

\begin{tabular}{|c|c|c|c|c|c|c|}
\hline \multirow[t]{2}{*}{ Espèce } & \multicolumn{2}{|c|}{ Densité } & \multicolumn{2}{|c|}{ Feuille } & \multicolumn{2}{|c|}{ Fruit } \\
\hline & Evolution & $\begin{array}{l}\text { FC } \\
(\%)\end{array}$ & Evolution & $\begin{array}{l}\text { FC } \\
(\%)\end{array}$ & Evolution & $\begin{array}{l}\text { FC } \\
(\%)\end{array}$ \\
\hline $\begin{array}{l}\text { Acacia } \\
\text { macrostachya }\end{array}$ & - & 11 & & & & \\
\hline $\begin{array}{l}\text { Acacia } \\
\text { raddiana }\end{array}$ & - & 22 & & & & \\
\hline $\begin{array}{l}\text { Acacia } \\
\text { senegal }\end{array}$ & - & 55 & & & & \\
\hline $\begin{array}{l}\text { Adansonia } \\
\text { digitata }\end{array}$ & - & 33 & + & 11,1 & + & 11,1 \\
\hline $\begin{array}{l}\text { Anogeissus } \\
\text { leiocarpus }\end{array}$ & - & 11 & & & & \\
\hline $\begin{array}{l}\text { Balanites } \\
\text { aegyptiaca }\end{array}$ & $-/+$ & $22 / 67$ & + & 77,8 & + & 77,8 \\
\hline $\begin{array}{l}\text { Boscia } \\
\text { senegalensis }\end{array}$ & - & 11 & + & 11,1 & + & 11,1 \\
\hline $\begin{array}{l}\text { Calotropis } \\
\text { procera }\end{array}$ & - & 22 & + & 11,1 & + & 11,1 \\
\hline $\begin{array}{l}\text { Combretum } \\
\text { aculeatum }\end{array}$ & - & 11 & & & & \\
\hline $\begin{array}{l}\text { Grewia } \\
\text { bicolor }\end{array}$ & - & 55 & & & & \\
\hline $\begin{array}{l}\text { Guiera } \\
\text { senegalensis }\end{array}$ & - & 33 & & & & \\
\hline Lannea acida & - & 11 & & & & \\
\hline $\begin{array}{l}\text { Piliostigma } \\
\text { thonningii }\end{array}$ & - & 11 & & & & \\
\hline $\begin{array}{l}\text { Sclerocarya } \\
\text { birrea }\end{array}$ & $-/+$ & $22 / 11$ & + & 33,8 & + & 33,3 \\
\hline $\begin{array}{l}\text { Terminalia } \\
\text { avicennioides }\end{array}$ & - & 11 & & & & \\
\hline $\begin{array}{l}\text { Ziziphus } \\
\text { mauritiana }\end{array}$ & - & 11 & & & & \\
\hline
\end{tabular}

FC : fréquence de citation ; - en diminution ; + en augmentation 


\section{Tableau VII}

Evolution de la densité et de la production de feuilles et de fruits selon les espèces à Téssékéré, Sénégal (1973 à 2010)

\begin{tabular}{|c|c|c|c|c|c|c|}
\hline \multirow[t]{2}{*}{ Espèce } & \multicolumn{2}{|c|}{ Densité } & \multicolumn{2}{|c|}{ Feuille } & \multicolumn{2}{|c|}{ Fruit } \\
\hline & Evolution & $\begin{array}{l}\text { FC } \\
(\%)\end{array}$ & Evolution & $\begin{array}{l}\text { FC } \\
(\%)\end{array}$ & Evolution & $\begin{array}{c}\text { FC } \\
(\%)\end{array}$ \\
\hline $\begin{array}{l}\text { Acacia } \\
\text { senegal }\end{array}$ & - & 16,7 & & & & \\
\hline $\begin{array}{l}\text { Adansonia } \\
\text { digitata }\end{array}$ & - & 33,3 & + & 33,3 & + & 33,3 \\
\hline $\begin{array}{l}\text { Balanites } \\
\text { aegyptiaca }\end{array}$ & $-/+$ & $\begin{array}{c}50 / \\
33,3\end{array}$ & + & 66,7 & + & 66,7 \\
\hline $\begin{array}{l}\text { Boscia } \\
\text { senegalensis }\end{array}$ & & & + & 16,7 & + & 16,7 \\
\hline $\begin{array}{l}\text { Calotropis } \\
\text { procera }\end{array}$ & $-/+$ & $\begin{array}{l}16,7 / \\
16,7\end{array}$ & & & & \\
\hline $\begin{array}{l}\text { Cissus } \\
\text { populnea }\end{array}$ & - & 16,7 & & & & \\
\hline $\begin{array}{l}\text { Combretum } \\
\text { aculeatum }\end{array}$ & - & 16,7 & & & & \\
\hline $\begin{array}{l}\text { Commiphora } \\
\text { africana }\end{array}$ & - & 16,7 & & & & \\
\hline $\begin{array}{l}\text { Feretia } \\
\text { apodanthera }\end{array}$ & - & 16,7 & & & & \\
\hline $\begin{array}{l}\text { Grewia } \\
\text { bicolor }\end{array}$ & - & 50 & & & & \\
\hline $\begin{array}{l}\text { Guiera } \\
\text { senegalensis }\end{array}$ & - & 16,7 & & & & \\
\hline $\begin{array}{l}\text { Maytenus } \\
\text { senegalensis }\end{array}$ & - & 33,3 & & & & \\
\hline $\begin{array}{l}\text { Sclerocarya } \\
\text { birrea }\end{array}$ & - & 83,3 & + & 33,3 & + & 33,3 \\
\hline $\begin{array}{l}\text { Sterculia } \\
\text { setigera }\end{array}$ & - & 16,7 & & & & \\
\hline $\begin{array}{l}\text { Terminalia } \\
\text { avicennioides }\end{array}$ & - & 16,7 & & & & \\
\hline $\begin{array}{l}\text { Ziziphus } \\
\text { mauritiana }\end{array}$ & - & 16,7 & & & & \\
\hline
\end{tabular}

FC : fréquence de citation ; - en diminution ; + en augmentation

Les résultats de l'enquête ont révélé une augmentation de la biomasse foliaire et fruitière de Ac. raddiana, Ac. seyal, Ad. digitata, Ba. aegyptiaca, Bo. senegalensis, Ca. procera, Co. micranthum, Faidherbia albida, Gr. bicolor, Sc. birrea, Zi. mauritiana à l'exception de Gr. bicolor, Co. micranthum et Ac. senegal. Les espèces les plus citées en termes de production foliaire ont été Ba. aegyptiaca $(65 \%)$ et Sc. birrea $(35 \%)$.

\section{DISCUSSION}

Les résultats de l'enquête confirment l'importance des espèces ligneuses pour les populations locales. Elles entrent dans l'alimentation humaine avec l'utilisation des feuilles et des fruits. Ces résultats concordent avec ceux de Agali (2009), Ouedrago (2008), Soubeiga (2004), Codja et al. (2003), Savy (2002), Ambé (2000), et Okafor (1991). Les espèces les plus consommées étaient des essences fruitières avec Ba. aegyptiaca, Ad. digitata et Zi. mauritiana. La consommation d'une espèce dépendrait de sa disponibilité (Niang, 2009).

\section{Tableau VIII}

Evolution de la densité et de la production de feuilles et de fruits selon les espèces à Widou, Sénégal (1973 à 2010)

\begin{tabular}{|c|c|c|c|c|c|c|}
\hline \multirow[t]{2}{*}{ Espèce } & \multicolumn{2}{|c|}{ Densité } & \multicolumn{2}{|c|}{ Feuille } & \multicolumn{2}{|c|}{ Fruit } \\
\hline & Evolution & $\begin{array}{l}\text { FC } \\
(\%)\end{array}$ & Evolution & $\begin{array}{l}\text { FC } \\
(\%)\end{array}$ & Evolution & $\begin{array}{l}\text { FC } \\
(\%)\end{array}$ \\
\hline $\begin{array}{l}\text { Acacia } \\
\text { raddiana }\end{array}$ & $-/+$ & $15 / 5$ & + & 10 & + & 5 \\
\hline Acacia senegal & - & 25 & + & 5 & & \\
\hline Acacia seyal & - & 5 & & & + & 5 \\
\hline $\begin{array}{l}\text { Adansonia } \\
\text { digitata }\end{array}$ & $-/+$ & $10 / 5$ & + & 20 & + & 20 \\
\hline $\begin{array}{l}\text { Balanites } \\
\text { aegyptiaca }\end{array}$ & $-/+$ & $15 / 65$ & + & 65 & + & 65 \\
\hline $\begin{array}{l}\text { Boscia } \\
\text { senegalensis }\end{array}$ & $-/+$ & $5 / 10$ & + & 10 & + & 10 \\
\hline $\begin{array}{l}\text { Calotropis } \\
\text { procera }\end{array}$ & $-/+$ & $10 / 5$ & + & 10 & + & 10 \\
\hline $\begin{array}{l}\text { Combretum } \\
\text { aculeatum }\end{array}$ & $-/+$ & $10 / 5$ & & & & \\
\hline $\begin{array}{l}\text { Combretum } \\
\text { micranthum }\end{array}$ & & & + & 5 & & \\
\hline $\begin{array}{l}\text { Commiphora } \\
\text { africana }\end{array}$ & - & 15 & & & & \\
\hline $\begin{array}{l}\text { Faidherbia } \\
\text { albida }\end{array}$ & & & + & 5 & + & 5 \\
\hline $\begin{array}{l}\text { Feretia } \\
\text { apodanthera }\end{array}$ & - & 5 & & & & \\
\hline Grewia bicolor & - & 35 & + & 5 & & \\
\hline $\begin{array}{l}\text { Guiera } \\
\text { senegalensis }\end{array}$ & - & 10 & & & & \\
\hline $\begin{array}{l}\text { Maytenus } \\
\text { senegalensis }\end{array}$ & - & 10 & & & & \\
\hline $\begin{array}{l}\text { Sclerocarya } \\
\text { birrea }\end{array}$ & $-/+$ & $45 / 15$ & + & 35 & + & 35 \\
\hline $\begin{array}{l}\text { Sterculia } \\
\text { setigera }\end{array}$ & - & 15 & & & & \\
\hline $\begin{array}{l}\text { Ziziphus } \\
\text { mauritiana }\end{array}$ & - & 5 & + & 5 & + & 5 \\
\hline
\end{tabular}

Les espèces ligneuses étaient aussi une source de fourrage dans l'alimentation du bétail. Elles constituaient la principale ressource fourragère pendant la longue saison sèche et surtout de soudure, ce qui était à l'origine de pratiques comme l'élagage et l'abaissement des branches.

En plus de la fonction alimentaire, les espèces ligneuses sont également utilisées dans la pharmacopée traditionnelle. En effet, « les plantes médicinales constituent des ressources précieuses pour la grande majorité des populations rurales en Afrique, où plus de $80 \%$ de cette population s'en sert pour assurer les soins de santé » (Nacoulma-Ouedraogo, 1996). Les éleveurs disposent de connaissances empiriques transmises de père en fils. Ils utilisent les arbres seuls ou avec des incantations pour guérir des maladies humaines.

Le mot garab en wolof (langue nationale du Sénégal) signifie arbre mais aussi médicament, ce qui conforte les propos d'un éleveur : « chaque arbre est un médicament, il suffit de connaître la partie de la plante à utiliser et les incantations à réciter ». Ce savoir ancien est 
généralement légué par les ascendants (Hadonou-Yovo et al., 2019) et porte surtout sur les connaissances des vertus des plantes servant par exemple d'intrants ou de recettes en médecine traditionnelle (Zabouh, 2014). Quatorze espèces végétales étaient utilisées comme plantes médicinales par les éleveurs pour le traitement de plusieurs pathologies, dont l'hypertension artérielle, le diabète, les problèmes gastriques, la diarrhée, le mal de dents.

Les ressources ligneuses étaient aussi utilisées dans d'autres domaines comme l'artisanat, les coutumes et traditions (mariage, circoncision, baptême). Les différentes utilisations des espèces ligneuses font partie des mécanismes d'adaptation des populations locales qui sont vulnérables aux conditions d'aridité et de pauvreté. L'accès à ces ressources se fait par un certain nombre de pratiques parfois destructrices à moyen ou long terme. Beaucoup d'éleveurs (45,7 \%), conscients de l'effet de telles pratiques utilisaient rarement les arbres surtout pour la construction. Aujourd'hui les populations locales sont conscientes de l'impact de ces pratiques sur le renouvellement de la ressource donc sur sa pérennité. Cette prise de conscience associée à une certaine crainte de l'autorité étatique (Service des eaux et forêts) a permis l'abandon de pratiques abusives dont la coupe des arbres. L'abondance de maisons construites à base de ciment dans la zone est une preuve de la diminution ou de l'abandon de l'utilisation du bois. Ces résultats diffèrent de ceux de Lykke et al. (2004) qui révèlent que l'habitat de type traditionnel reste étroitement lié aux ressources naturelles végétales qui fournissent l'essentiel des matériaux de construction (piquets, perches, traverses, supports, paille, chaume, cordes). La perception des populations sur l'état des ressources a montré que les éleveurs maîtrisaient bien leur milieu. Cependant la perception variait d'un éleveur à un autre car elle reposait sur le pâturage qui était exploité par le troupeau.

Par rapport à la dynamique de la végétation du Ferlo, les éleveurs avaient une idée précise de l'état actuel de la ressource, de son évolution dans le temps et des facteurs d'évolution. Pour eux la dynamique de la végétation était fortement tributaire de la pluviométrie (Diouf, 2002). Ils étaient également convaincus que le sol était de très bonne qualité et que la régénération et la production dépendaient uniquement de la pluie.

Ces dernières années la bonne pluviométrie a entraîné une amélioration de la quantité de fourrage surtout herbacé. Des rejets de ligneux (Grewia bicolor, Commiphora africana) appétés par le bétail ont été observés dans la localité (Bakhoum, 2013). Même si avec la longue période sèche ces rejets n'arrivent pas à survivre, ils témoignent de la disponibilité de leurs semences au niveau du stock semencier et de l'importance de l'eau pour leur germination et leur épanouissement.

\section{Remerciements}

Nous remercions le Pôle pastoralisme et zones sèches (PPZS) pour l'appui logistique et financier pour la collecte de données dans le cadre du projet ECLIS.

\section{Déclaration des contributions des auteurs}

$\mathrm{AB}$ a réalisé le guide d'entretien, collecté les données sur le terrain et rédigé le document. OS a participé aux enquêtes sur le terrain et a révisé le document. $\mathrm{DN}$ et $\mathrm{SD}$ ont révisé le manuscrit. $\mathrm{AI}$ a participé à la conception du guide d'entretien et à la révision du document.

\section{Conflits d'intérêts}

L'étude a été réalisée sans aucun conflit d'intérêts.

\section{REFERENCES}

Agali A.B., 2009. Diversité, structure et perceptions locales des espèces ligneuses fourragères dans le terroir de Torodi, Ouest Niger. Mémoire de DEA en Biologie et Ecologie végétales. Université d'Ouagadougou, Burkina Faso, 48p.

Ambé G.A., 2000. Les fruits sauvages comestibles des savanes guinéennes de la Côte d'Ivoire, état de connaissance par une population locale, les Malinkés. Biotech. Agron. Soc. Environ. 5 (1) : 43-48

Bakhoum A., 2013. Dynamique des ressources fourragères: indicateur de résilience des parcours communautaires de Téssékéré au Ferlo (Nord-Sénégal). Thèse Doct., Université Cheikh Anta Diop, Dakar, Sénégal, 115 p.

Banoin M., Jouve P., 2000. Déterminants des pratiques de transhumance en zone agro-pastorale sahélienne cas de I'arrondissement de Mayahi, au Niger. In : Bourbouze A. (ed.), Qarro M. (ed.). Rupture : nouveaux enjeux nouvelles fonctions, nouvelle image de l'élevage sur parcours. Montpellier, France : CIHEAM, p. 91-105. (Options Méditerranéennes : Série A. Séminaires Méditerranéens; n. 39). Séminaire International du Réseau Parcours. El Jadida, Maroc16-18 Avril 1998,. http://om.ciheam.org/om/pdf/ a39/CI000350.pdf

Behnke R.H., Scoones I., Kerven C., 1993. - Range Ecology at Disequilibrium. ODI, IIED, London 248 p.

Betti J.L., 2001.Vulnérabilité des plantes utilisées comme antipaludiques dans l'arrondissement de Mintom au sud de la réserve de biosphère du Dja (Cameroun). National Botanic Garden of Belguim. Syst. Geogr. Plants, 71 (2): 661 - 678, doi: 10.2307/3668709

Codja J.T.C., Assogbadjo A.E., Ekue M.R.M., 2003. Diversité et valorisation au niveau local des ressources végétales forestières alimentaires du Bénin. Cah. Agric. 12 (5) : 321-331

Diouf M., 2002. Dynamique du peuplement ligneux d'une végétation sahélienne au Nord-Sénégal (Afrique de l'Ouest), J. Sci., vol. 2, (1), 1-9

Fontes J., Guinko S., 1995. Carte de la végétation et de l'occupation du sol du Burkina Faso. Notice explicative du Ministère de la coopération française. Projet Campus, Toulouse, France, 86 p.

Hadonou-Yovo A.G., Houessou L.G., Lougbegnon T.O., Adebi Y., Sinasson G.K.S., Semevo D.F., Lange U., et al., 2019. Diversité et formes d'utilisation des espèces ligneuses de la Réserve de biosphère du Mono (Bénin), VertigO - la revue électronique en sciences de l'environnement, volume 19 (2), consulté le 10 mars 2020. URL : https://om.ciheam.org/om/pdf/ a39/CI000350.pdf; DOI : 10.4000/vertigo.26257

Lykke A.M., 2000. Local perceptions of vegetation change and priorities for conservation of woody-savanna vegetation in Senegal, J. Environ. Manag., 59: 107120, doi: 10.1006/jema.2000.0336

Lykke A.M., Kristensen M., Ganaba S., 2004. Valuation of local use and dynamics of 56 species in the Sahel. Biodivers. Conserv., 13: 1961-1990, doi: 10.1023/B:BIOC.0000035876.39587.1a

Nacoulma-Ouedraogo O., 1996. Les plantes médicinales et les pratiques médicales traditionnelles au Burkina Faso. Cas du plateau central. Thèse ès Sc. Nat. FAST/UO Tome 2257 pages

Niang K., 2009. L'arbre dans les parcours communautaires du Ferlo-Nord (Sénégal), Mémoire de DEA, Faculté des Sciences et Techniques, Université Cheikh Anta Diop, Dakar, Sénégal,67 p.

Okafor J.-C., 1991. Amélioration des essences forestières donnant des fruits comestibles. Unasylva 42 (165): 1-10

Ouedraogo I., 2008. Diversité des espèces ligneuses utiles de la région du Nord du Burkina Faso ; état des peuplements de cinq espèces d'importance socio-économique. Mémoire d'Ingénieur en Eaux et Forêts. Université de Bobo Dioulasso, Burkina Faso, 68 p.

Sarr O., Diatta S., Gueye M., N'diaye P.-M., Guisse A., Akpo L.-E., 2013. Importance des ligneux fourragers dans un système agropastoral au Sénégal (Afrique de l'Ouest), Rev. Méd. Vét., Ecole Nationale Vétérinaire de Toulouse. vol. 164 (1) : 2-8, hal-01722601

Savy M., 2002. Diversité, variété alimentaire et état nutritionnel des mères de jeunes enfants en milieu rural défavorisé. Mémoire DEA., Université de Ouagadougou Burkina Faso et Université Pierre et Marie Curie Paris France, $34 \mathrm{p}$.

Soubeiga K.-J., 2004. Analyse de la demande des produits forestiers non ligneux dans l'alimentation des ménages ruraux : cas des départements de Bondokuy (Mouhoun) et de Niandialia (Boulkiemdé). Mémoire d'Ingénieur, Université Polytechnique de Bobo-Dioulasso, Burkina Faso, 57p.

Traoré L., 2008. Inventaire des espèces ligneuses utilitaires de la région SudOuest du Burkina Faso et état des populations de trois espèces à haute valeur économique. Mémoire DEA Université d'Ouagadougou, Burkina Faso, $50 \mathrm{p}$. 


\section{Summary}

Bakhoum A., Sarr O., Ngom D., Diatta S., Ickowicz A. Woody fodder uses and pastoral practices in the rural community of Tessekere, Ferlo, Northern Senegal

The rural community of Téssékéré is 97\% Peul with extensive livestock farming as its main activity. Transhumance is practiced in this area over small or long distances depending on the availability of fodder. Surveys conducted among loca herders have made it possible to identify their pastoral practices and to collect their perception of the current state of the vegetation and its evolution, and the factors responsible for this state. Trees are used in this community for food (94.3\%), feed $(94.3 \%)$, pharmacopeia $(82.8 \%)$, and domestic uses such as firewood (28.6\%) and timber $(37.14 \%)$. In order to access the different parts of the trees, farmers often practice cutting or pruning. The most used species are Balanites aegyptiaca $(14.3 \%$ to $88.6 \%)$, Calotropis procera $(14.3 \%$ to $28.6 \%)$, Sclerocarya birrea (8.6\% to $25.7 \%)$, Adansonia digitata $(8.6 \%$ to $20 \%$ ) depending on the area and the needs. The majority of herders $(51.4 \%)$, now aware of the impact of these practices on the woody stands, have given up cutting and instead lower branches to feed their livestock.

Keywords: tree, multiple use, forage, pastoralists, surveys, climate change, Senegal

\section{Resumen}

Bakhoum A., Sarr O., Ngom D., Diatta S., Ickowicz A. Uso de forrajes leñosos y prácticas pastorales en la comunidad rural de Téssékéré, Ferlo (norte de Senegal)

La comunidad rural de Téssékéré es 97\% peul, con una actividad principal de cría extensiva. La trashumancia se practica en esta zona, sobre pequeñas o grandes distancias, en función de la disponibilidad del forraje. Las encuestas realizadas con criadores locales permitieron la identificación de sus prácticas pastorales, así como recoger su percepción sobre el estado actual de la vegetación, su evolución, y los factores responsables de este estado. El árbol es utilizado en esta comunidad para la alimentación humana $(94,3 \%$ ) y animal $(94,3 \%)$, en la farmacopea $(82,8 \%)$ y para usos domésticos como madera de servicio $(28,6 \%)$ y de construcción $(37,14 \%)$. Para acceder a las diferentes partes de los árboles, los criadores practican a menudo la tala o la poda. Las especies más explotadas son Balanites aegyptiaca $(14,3 \%$ a $88,6 \%)$, Calotropis procera $(14,3 \%$ a $28,6 \%)$, Sclerocarya birrea $(8,6 \%$ a $25,7 \%)$, Adansonia digitata $(8,6 \%$ a $20 \%)$ según áreas y necesidades. La mayoría de los agricultores (51,4\%), conscientes hoy del impacto de estas practicas sobre el suministro leñoso, han abandonado la tala y practican el descenso de las ramas para alimentar el ganado.

Palabras clave: árboles, uso múltiple, forrajes, pastoralistas, encuestas, cambio climático, Senegal 